\title{
ON INVARIANT MEASURES
}

\author{
BY DONALD S. ORNSTEIN \\ Communicated by P. R. Halmos, April 13, 1960
}

Given a measurable transformation on a measure space one can ask whether or not there is an equivalent measure that is invariant under the transformation. This problem is discussed very thoroughly in Halmos' Lectures on ergodic theory, pp. 81-90, 97. The first result along these lines is due to E. Hopf who obtained necessary and sufficient conditions for the existence of a finite invariant measure. The condition is that the whole space is "bounded," i.e. that the space is not a "copy" of a subset of strictly smaller measure. ("Copy" is defined below.) Recently Hajian and Kakutani (the paper is not yet published) showed that Hopf's condition is equivalent to the nonexistence of a set of nonzero measure having infinitely many disjoint images under the powers of the transformation. In [3] Halmos proved that there was a sigma-finite invariant measure if and only if the space was the union of a countable number of "bounded" sets. It was not known however whether or not every transformation had this property. Our example shows that there are transformations that admit no equivalent invariant measures.

THEOREM. There exists a 1-1 invertible measurable and nonsingular transformation, $T$, on the unit interval such that there is no sigma-finite measure equivalent to Lebesgue measure which is invariant under $T$.

We could modify the example a little so that the only invariant measure which is absolutely continuous with respect to Lebesgue measure is identically 0 .

(A 1-1 invertible, measurable, nonsingular transformation is one such that it and its inverse take measurable sets into measurable sets and sets of measure 0 into sets of measure 0 . A measure equivalent to Lebesgue measure is a measure which is defined on the same class of measurable sets and has the same sets of measure 0 . Sigmafinite means that the interval is the union of a countable number of sets of finite measure.

It is possible to have a sigma-finite measure equivalent to Lebesgue measure such that every interval has infinite measure and it is this sort of thing that complicates our construction.)

A transformation $T$ on the unit interval will be said to have property $P$ if: for any integer $N$ and any set $S$ of Lebesgue measure $>9 / 10$ there is a set $M C S$ of Lebesgue measure $1 / 8$ such that there 
are $N$ disjoint "copies" of $M$ in $S$. [The set $A$ is said to be a "copy" of the set $B$ if $A$ and $B$ are each the union of a countable number of disjoint measurable subsets $A_{i}$ and $B_{i}$ respectively, and $A_{i}=T^{n_{i}}\left(B_{i}\right)$ where the $n_{i}$ are integers, which might be positive, negative or 0 .]

A transformation $T$ with property $P$ can have no sigma-finite invariant measure equivalent to Lebesgue measure. To see this note that if there were such an invariant measure $L^{\prime}$ there would be a set $S$ of Lebesgue measure $>9 / 10$ which has finite $L^{\prime}$ measure. Our property implies that $S$ contains sets of Lebesgue measure $1 / 8$ with arbitrarily small $L^{\prime}$ measure. This is impossible (see $[2$, p. 125, Theorem B]).

Construction of $T$. We will do this in stages, each time defining $T$ on a larger set and never changing $T$ after it has already been defined. At the $N$ th stage we will have the interval $I$ (except for the left end point) written as the union of $K_{N}$ disjoint intervals (all our intervals will contain their right end point but not their left) $I_{j}^{N}\left(j=1, \cdots, K_{N}\right)$ (not necessarily the same length) and $T$ will map $I_{j}^{N}$ linearly onto $I_{j+1}^{N}\left(1 \leqq j \leqq K_{N}-1\right) . T$ will not be defined on $I_{K_{N}}^{N}$ and $T^{-1}$ will not be defined on $I_{1}^{N}$.

We will now define $T$ on part of $I_{K_{N}}^{N}$ (more than $1 / 2$ of it) and $T^{-1}$ on part of $I_{1}^{N}\left(1 / 2\right.$ of $\left.I_{1}^{N}\right)$. We will denote by $I_{1,1}^{N}$ the left half of $I_{1}^{N}$. We will divide the rest of $I_{1}^{N}$ into $K_{1}^{N}-1$ disjoint equal intervals $I_{1, j}^{N}\left(2 \leqq j \leqq K_{N}^{1}\right)$. ( $K_{N}^{1}$ will be any integer $>1$ and large enough so that $N \cdot K_{N} \cdot L\left(T^{i}\left(I_{1, j}^{N}\right)\right)<1 / 100$ for any $0 \leqq i \leqq K_{N}-1$ and any $2 \leqq j \leqq K_{N}^{1}$. $(L(A)=$ Lebesgue measure of the set $A$.)

We now have $I_{K_{N}}^{N}$ divided up into disjoint intervals $T^{K_{N}-1}\left(I_{1, j}^{N}\right)$ $\left(1 \leqq j \leqq K_{N}^{1}\right)$. We will now define $T$ on $\cup_{j=1}^{K_{N}{ }^{1}-1} T^{K_{N}-1}\left(I_{1, j}\right)$ (and, automatically, $T^{-1}$ on $\left.\bigcup_{j=2}^{K_{N}^{1} N} I_{1, j}^{N}\right)$ by sending $T^{K_{N}-1}\left(I_{1, j}^{N}\right)$ linearly onto $I_{1, j+1}^{N}$.

We are now back in our original situation. $I$ is broken up into $K_{N}^{1} K_{N}=K_{N+1}$ disjoint intervals $I_{l}^{N+1}\left(1 \leqq l \leqq K_{N+1}\right)$ where

$$
T^{k}\left(I_{1, j}^{N}\right)=I_{K_{N}(j-1)+k+1}^{N+1} \quad\left(0 \leqq k \leqq K_{N}\right),\left(1 \leqq j \leqq K_{N}^{1}\right) .
$$

$T$ maps $I_{l}^{N+1}$ linearly onto $I_{l+1}^{N+1}\left(1 \leqq l \leqq K_{N+1}\right)$.

Choose the intervals $I_{1}^{1}=(1 / 2,1]$ and $I_{2}^{1}=(0,1 / 2]$. This finishes the construction

$$
L\left(I_{K_{N}+1}^{N+1}\right) \leqq \frac{1}{2} L\left(I_{K_{N}}^{N}\right) \quad \text { and } \quad L\left(I_{1}^{N+1}\right)=\frac{1}{2} L\left(I_{1}^{N}\right) .
$$

This means that the measure of the sets on which $T$ and $T^{-1}$ are not defined is tending to 0 and hence we have defined $T$ and $T^{-1}$ on all 
of $I$ except a set of measure 0 (in fact we define it on all points except 0 and 1). It is easy to check that $T$ and $T^{-1}$ are measurable. Both are nonsingular because any set of non 0 -measure must, at some stage of the construction, intersect some interval other than the two end ones in a set $Q$ of non 0 -measure, and $T(Q)$ and $T^{-1}(Q)$ must have non 0 measure.

Proof that $T$ has property $P$. Let $S$ be a set of measure $>9 / 10$ and let $N$ be an integer. Let $I_{k}^{N+1}=J_{k}$. It is easy to see that we can divide each $J_{i}$ into $2^{K_{N+1}}$ disjoint measurable subsets, $J_{i}^{l}\left(1 \leqq l \leqq 2^{K_{N+1}}\right)$ such that each $J_{l}^{l}$ lies entirely in either $S$ or the complement of $S$ and $T\left(J_{\imath}^{l}\right)=J_{i+1}^{l}\left(1 \leqq i \leqq K_{N+1}-1\right),\left(1 \leqq l \leqq 2^{K_{N+1}}\right)$ [we will show how to do this, i.e. how to define the set $J_{i}^{l}$. Look at the dyadic expansion of $l$. Denote the $r$ th term by $(l, r)$ and denote $J_{i} \cap S$ by ${ }^{0} J_{i}$ and $\left(J_{i}-{ }^{0} J_{i}\right)$ by ${ }^{1} J_{i}$. Now let

$$
J_{i}^{l}=\bigcap_{j=1}^{K_{N+1}} T^{i-j}\left({ }^{(l, j)} J_{j}\right) \quad\left(1 \leqq i \leqq K_{N+1}\right) \quad\left(1 \leqq l \leqq 2^{K_{N+1}}\right) .
$$

We then have $\left.T\left(J_{i}^{l}\right)=\bigcap_{j=1}^{K_{N+1}} T^{i+1-j\left({ }^{(l, j)}\right.} J_{j}\right)=J_{i+1}^{l}$ and $\left.J_{i}^{l} \subset^{(l, i)} J_{i}\right]$.

Define $B^{l}=\bigcup_{i=1}^{K_{N+1}} J_{i}^{l}$. Let $u_{l}$ be the measure of $B^{l}$. If there are $N K_{N}$ or more intervals $J_{k}^{l}\left(K_{N} \leqq k \leqq K_{N+1}\right)$ in $S$ we will call $B^{l}$ a good $B^{l}$.

$$
\frac{L\left(J_{i}^{l}\right)}{L\left(J_{i}\right)}=\frac{L\left(J_{k}^{l}\right)}{L\left(J_{k}\right)}=u_{l}, \quad\left(1 \leqq k \leqq i \leqq K_{N+1}\right),
$$

(1) is true because $J_{i}=T^{i-k}\left(J_{k}\right)$ and $J_{i}^{l}=T^{i-1}\left(J_{k}^{l}\right)$ and $T^{i-k}$ maps $J_{k}$ linearly onto $J_{i}$.

$$
\sum_{i=1}^{K_{N}} L\left(J_{i}\right)=\sum_{k=0}^{K_{N}-1} L\left(T^{k}\left(I_{1,1}^{N}\right)\right)=\frac{1}{2}
$$

because $I_{1,1}^{N}$ was chosen to be the left half of $I_{1}^{N}$.

(3) If $B^{l}$ is not a good $B^{l}$ then the complement of $S$ intersects $B^{l}$ in a set of measure $\geqq(1 / 2-1 / 100) u_{l}$.

To see (2) we note first that the sum of the measures of any $N K_{N}$ of the $J_{i}\left(K_{N}<i \leqq K_{N+1}\right)$ is less than $1 / 100$ because of our choice of $K_{N}^{1}$. It now follows from (1) that for a fixed $l$ the sum of the measures of any $N K_{N}$ of the $J_{i}^{l}\left(K_{N}<i \leqq K_{N+1}\right)$ is less than $u_{l} / 100$.

From (2) we get that $\sum_{i=K_{N+1}}^{K_{N+1}} L\left(J_{i}\right)=1 / 2$ and this together with (1) implies that $\sum_{i=K_{N+1}}^{K_{N+1}} L\left(J_{i}^{l}\right)=u_{l} / 2$. This and the last line of the preceding paragraph give (3).

(4) The sum of the measures of the good $B^{l}$ is greater than $1 / 2$. 
This follows from (3) and the fact that the complement of $S$ has measure less than $1 / 10$.

Let $M^{1}$ be the part of $\bigcup_{i=1}^{K_{N}} J_{i}$ which lies in the good $B^{l}$.

$$
L\left(M^{1}\right) \geqq \frac{1}{4} \text {. }
$$

We get (4) in the following way: (2) and (1) imply $\sum_{i=1}^{K_{N}} L\left(J_{i}^{l}\right)$ $=u_{l} / 2$. This together with (4) implies (5).

Let $M$ be the part of $M^{1}$ that lies in $S . L(M)>1 / 4-1 / 10>1 / 8$. $M^{1}$, and therefore $M$, has $N$ disjoint copies in $S$ because $\bigcup_{i=1}^{K_{N}} J_{i}^{l}$ has $N$ disjoint copies in $S \cap B^{l}$ if $B^{l}$ is good.

$M$ is the subset of $S$ needed for property $P$.

\section{REFERENCES}

1. P. Halmos, Lectures in ergodic theory, Math. Society of Japan, 1956.

2. —, Measure theory, Van Nostrand, 1950.

3. - Ann. of Math. (1947) p. 735.

4. E. Hopf, Theory of measure and invariant integrals, Trans. Amer. Math. Soc. vol. 34 (1932) pp. 373-393.

UNIVERSITY OF WisCONSIN 